\title{
Anti-Developmental Effects of Imazosulfuron on Zebrafish Embryos During Development
}

\author{
Sunwoo Park ${ }^{1}$, Gwonhwa Song ${ }^{1, *}$ and Whasun Lim ${ }^{2, *}$ \\ ${ }^{1}$ Institute of Animal Molecular Biotechnology and Department of Biotechnology, College of Life Sciences and \\ Biotechnology, Korea University, Seoul 02841, Korea \\ ${ }^{2}$ Department of Food and Nutrition, College of Science and Technology, Kookmin University, Seoul 02707, Korea
}

Received February 28, 2020

Revised March 11, 2020

Accepted March 12, 2020

*Correspondence

Gwonhwa Song

E-mail: ghsong@korea.ac.kr

ORCID

https://orcid.org/0000-0003-2817-5323

Whasun Lim

E-mail: wlim@kookmin.ac.kr

ORCID

https://orcid.org/0000-0002-1328-0465
ABSTRACT Imazosulfuron is globally considered as a relatively safe herbicide that controls plant growth by interfering with amino acid synthesis. It is stable, persists in the soil, and has low toxicity; however, studies about the toxic effects of imazosulfuron on non-targeted aquatic vertebrates are scarce. In this study, imazosulfuron was able to induce acute lethality on zebrafish embryos within $48 \mathrm{~h}$. Imazosulfuron also had adverse effects on heartbeats and induced abnormal development with pericardial edema and scoliosis. Moreover, apoptosis and oxidative stress were increased by imazosulfuron in a dose-dependent manner. Thus, all our results showed that imazosulfuron has toxic effects on zebrafish embryogenesis.

Keywords: anti-development, apoptosis, herbicide, imazosulfuron, zebrafish embryo

\section{INTRODUCTION}

Sulfonylurea herbicides have been widely used in Europe and America since the 1970s due to their wide range of action, high selectivity, and low toxicity (Blair and Martin, 1988). Imazosulfuron is one of the sulfonylurea herbicides used for broad-leaved weeds and grass, and in crops, it exhibits high effectiveness even at low dosages (Sondhia, 2008). Once it is absorbed by foliage or roots, it interferes with amino acid synthesis and cell division, leading to growth inhibition in plants (Hay, 1990). Recently, resistance to sulfonylurea herbicides has been reported (Kuk et al., 2004). However, it is known that the solvent used for imazosulfuron determines its absorption and residual rates in the soil; for example, acetonitrile percentage is inversely proportional to imazosulfuron absorption rate in clay loam environments (Morrica et al., 2000). Imazosulfuron accumulation in the soil could also affect non-targeted bacteria; for example, sulfometuron methyl, another sulfonylurea herbicide, inhibited Salmonella typhimurium growth by interrupting acetolactate synthase activity (LaRossa and Schloss, 1984). In addition, residual sulfonylurea herbicides are only eliminated through three water treatment steps including coagulation, activated carbon absorption, and chlorine-based disinfection. However, sulfonylurea may have been transformed into more stable structures during the disinfection processes and subsequently dissolved in drinking water (Wang et al., 2015). Even with the risks associated with imazosulfuron usage, there is a lack of studies on the toxic effects of imazosulfuron in aquatic vertebrates.

Nowadays, mainstream toxicology focuses on the molecular changes due to various chemical treatments rather than the detection of endpoint effects (Villeneuve et al., 2014). As such, zebrafish has been used for studies involving molecular dynamics, real-time monitoring 
of external changes, and cellular changes. Large-scale genetic screening of zebrafish served as a big step in understanding the molecular functions during vertebrate development (Haffter et al., 1996). Currently, zebrafish is an emerging alternative vertebrate animal model; they are considered as a great model species because they are transparent, relatively easy to maintain, have short generation time, and grow fast (Lieschke and Currie, 2007). Their high fecundity also provides a larger sample size, which improves reliability of the studies. In addition, there is no need for invasive techniques or treatments because zebrafish automatically absorb external chemicals in the water (Garcia et al., 2016). Furthermore, a lot of zebrafish genes and the patterns they exhibit during development are conserved in vertebrates. Therefore, the anatomical and physiological characteristics of the zebrafish are very similar to those of humans (Pickart and Klee, 2014). Due to these advantages, various studies have utilized the zebrafish model to evaluate heart, kidney, liver, immune system, and neuron-related toxicity (Lieschke and Currie, 2007). In previous studies, zebrafish showed morphological and behavioral abnormalities in response to commonly used herbicides including ametryn, 2,4-dichlorophenoxyacetic acid, and metamifop (Li et al., 2017; Moura et al., 2018). However, there is no toxicological study on the effects of imazosulfuron on zebrafish embryos.

In this study, we investigated the toxic effects of imazosulfuron on zebrafish embryos by detecting morphological and intracellular changes it caused. The specific objectives of this study were to: 1) provide the toxicity information of imazosulfuron on aquatic invertebrates; 2 ) detect the pathological changes of zebrafish embryos in response to imazosulfuron; and 3) discover the mechanisms behind zebrafish death due to imazosulfuron. This study and its results provide the first step in elucidating the toxicological effects of imazosulfuron in aquatic vertebrates.

\section{MATERIALS AND METHODS}

\section{Zebrafish model and morphology}

Wild-type zebrafish was obtained from the Zebrafish Center for Disease Modeling (KNRRC, Chungnam National University, Republic of Korea). We housed the zebrafish following the guidelines of Korea University, which required the temperature to be set at $28.5^{\circ} \mathrm{C}$ with $12-\mathrm{h}$ dark/light cycles. Imazosulfuron (Cat No. 32919, Sigma Aldrich, USA) was incubated with zebrafish embryos until $48 \mathrm{hpf}$ for all assays. Imazosulfuron was diluted in $0.003 \%$ phenylthiourea (PTU), which was used to inhibit the zebrafish pigmentation. Viability, heartbeat, and zebrafish morphologies were detected using the LEICA DM 2500 (Leica, Germany) microscope. Pericardial edema was measured as boundary area and scoliosis were measured as angle between the ear and tail using ImageJ software (NIH, USA).

\section{Detection of apoptotic cells in zebrafish}

To detect apoptotic cells in zebrafish embryos, we stained imazosulfuron pre-treated zebrafish with acridine orange $(5 \mu \mathrm{g} / \mathrm{mL})$ for an additional $1 \mathrm{~h}$. After incubation, embryos were washed in fresh medium, anesthetized, and then placed on slides. The green fluorescence of apoptotic cells was detected using an upright fluorescence microscope (ZEISS, Germany).

\section{ROS production in zebrafish}

Reactive oxygen species (ROS) generation in imazosulfuron pre-treated embryos was quantified by staining the embryos with $20 \mu \mathrm{g} / \mathrm{mL}$ of dichloro-dihydro-fluorescein diacetate (DCFH-DA) green fluorescent dye for $1 \mathrm{~h}$. After incubation, embryos were washed, anesthetized, and placed on the slides. The green fluorescence of oxidized DCF was visualized using an upright fluorescence microscope (ZEISS, Germany). Both staining and fluorescence microscopy were performed in the dark. The intensity of each image was analyzed using ImageJ software (NIH, USA).

\section{Statistical analysis}

All data were evaluated using analysis of variance (ANOVA) reflecting the general linear model (PROC-GLM) of the SAS program (SAS Institute, Cary, NC, USA). Statistical significance was set as a value of $p<0.05$ between groups. All data are shown as mean \pm SEM.

\section{RESULTS}

\section{Anti-developmental effects of imazosulfuron on zebrafish embryos}

We observed the overall development of zebrafish em- 
bryos in response to imazosulfuron treatment (Fig. 1A) and observed that it did not change body length, eye size, or yolk sac (data not shown); however, imazosulfuron was observed to induce pericardial edema $(p<0.01)$ and scoliosis $(p<0.01)$ in zebrafish embryos (Fig. 1B and 1C).

\section{Lethal effects of imazosulfuron on zebrafish embryos}

To investigate the lethal effects of imazosulfuron on zebrafish embryos, we treated embryos with various concentrations of imazosulfuron for $48 \mathrm{~h}$. Imazosulfuron gradually decreased the viability of zebrafish embryos (Fig. 2A): imazosulfuron at a dosage of $200 \mu \mathrm{M}$ decreased zebrafish viability to $78.3 \%$ ( $p<0.01)$. From $20 \mu \mathrm{M}$, imazosulfuron also decreased the heartbeat of embryos (Fig. 2B). The average number of heartbeats was 118 beats per minute in $48 \mathrm{hpf}$ embryos, and it decreased to 53 beats per minute after $200 \mu \mathrm{M}$ imazosulfuron treatment $(p<$ 0.001). Apoptotic cells in the eyes and brain are shown as green dots due to acridine orange staining (Fig. 2C). Apoptotic cells were observed to increase in response to imazosulfuron treatment, especially in the frontal part of the embryos (Fig. 2D).

\section{Oxidative stress mediated apoptosis on zebrafish embryos}

To verify the mechanism of apoptosis in zebrafish embryos, we detected oxidative stress in response to imazosulfuron treatment. We measured ROS production, represented by green signals, in the embryos (Fig. 3A).
We therefore observed that imazosulfuron increased ROS production in the eyes and yolk sac of the zebrafish embryos (Fig. 3B). The results indicated that the generation of ROS was gradually increased by imazosulfuron in a dose-dependent manner and the ROS production especially increased in pericardial edema region of zebrafish embryos.

\section{DISCUSSION}

Imazosulfuron is a sulfonylurea herbicide that inhibits amino acid synthesis and plant development. It shows higher selectivity, effectiveness, and safety, and is considered as a new post-emergence herbicide (Morrica et al., 2001). According to the Pesticide Properties Database (PPDB) from the University of Hertfordshire, it takes more than 50 days for $50 \%$ of imazosulfuron to be degraded in the soil, and 120 days are needed for $50 \%$ degradation in sediment. Therefore, it has a high potential to affect aquatic vertebrates and invertebrates. However, until now, studies about the toxic effects and the mechanisms of imazosulfuron on aquatic organisms are lacking. In this study, we detected the anti-developmental effects of imazosulfuron on zebrafish embryos with heart dysfunction and scoliosis. Furthermore, imazosulfuron decreased the viability of embryos by ROS-related apoptosis.

During embryogenesis, heart formation has a role in blood circulation, nutrition, and energy delivery to growing cells. If heart development is damaged, several patho-

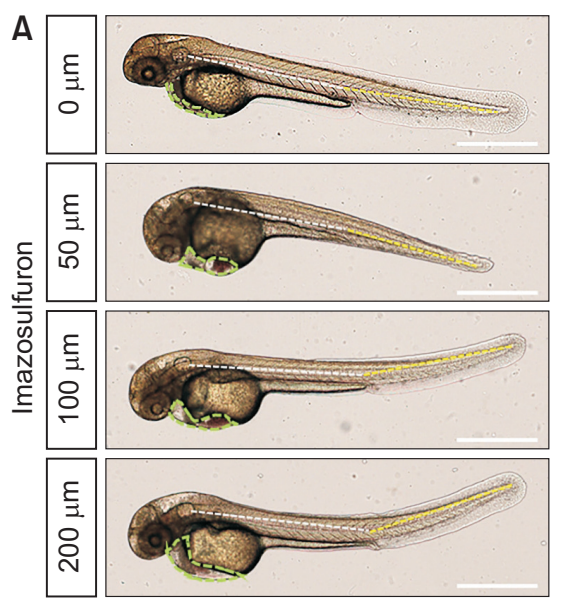

B

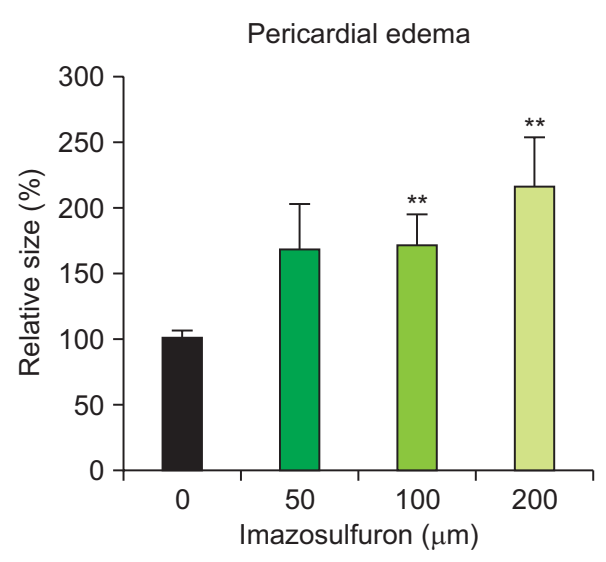

C

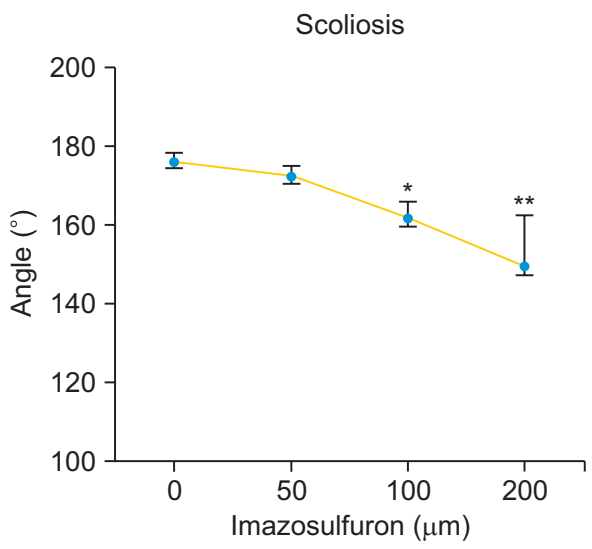

Fig. 1. The morphological changes of zebrafish embryos. (A) Abnormal development of zebrafish embryos was detected by LEICA DM 2500. Pericardial edema (B) and scoliosis (C) in response to the imazosulfuron treatment were detected by LEICA DM 2500 and analyzed by ImageJ software. Scale bar represents $0.5 \mathrm{~mm}$. For these experiments, 20 embryos were used per dosage $\left(^{*} p<0.05\right.$ and ${ }^{* *} p$ $<0.01)$. 
A

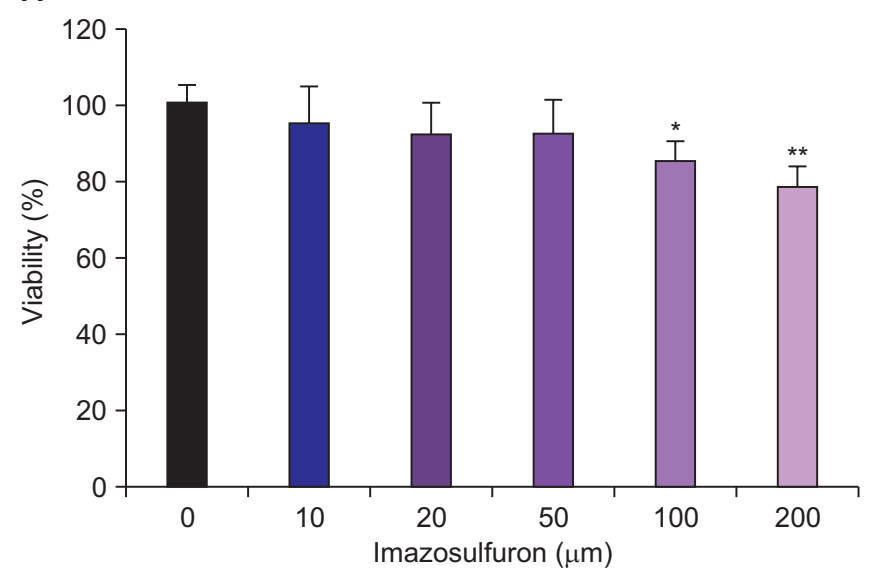

B

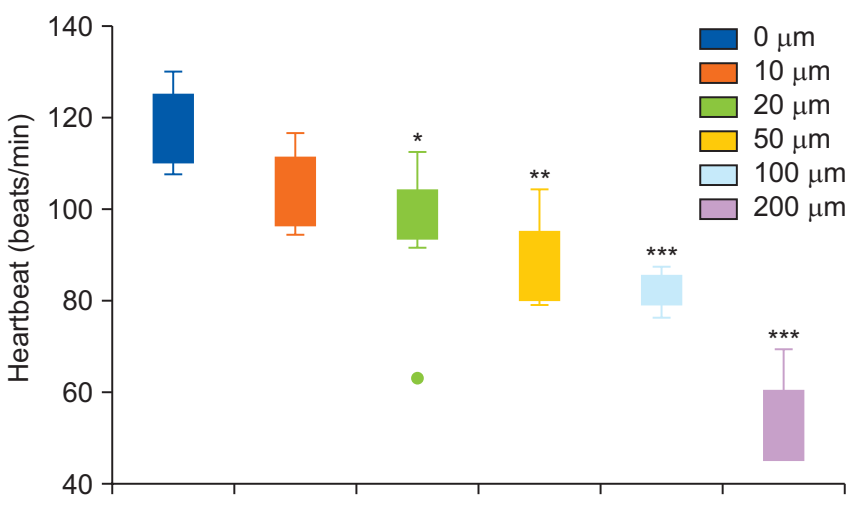

C

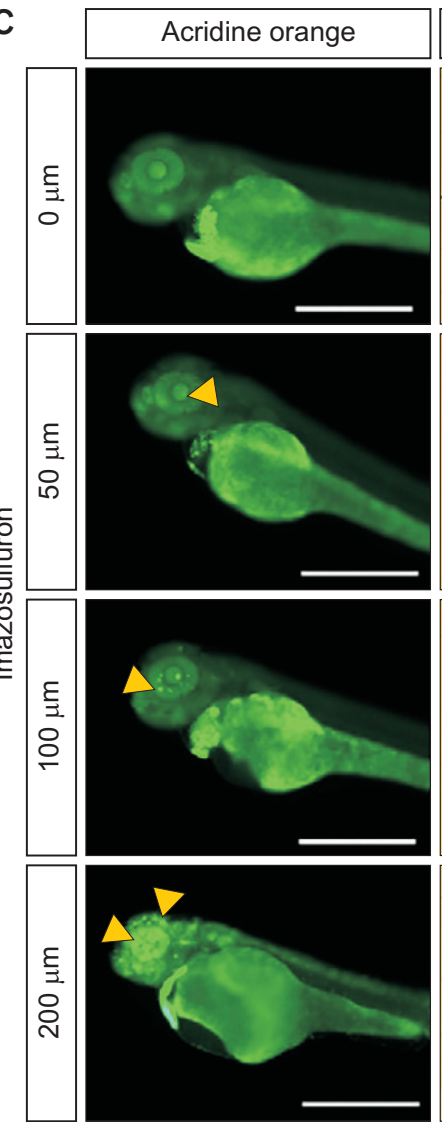

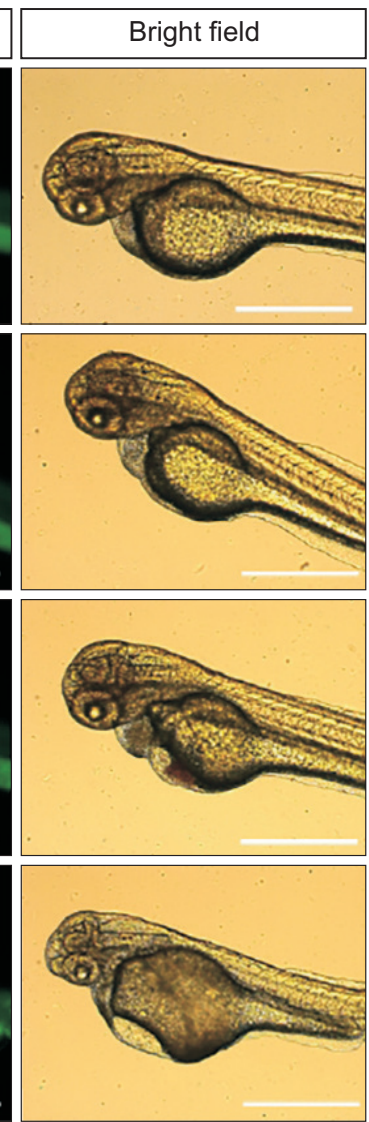

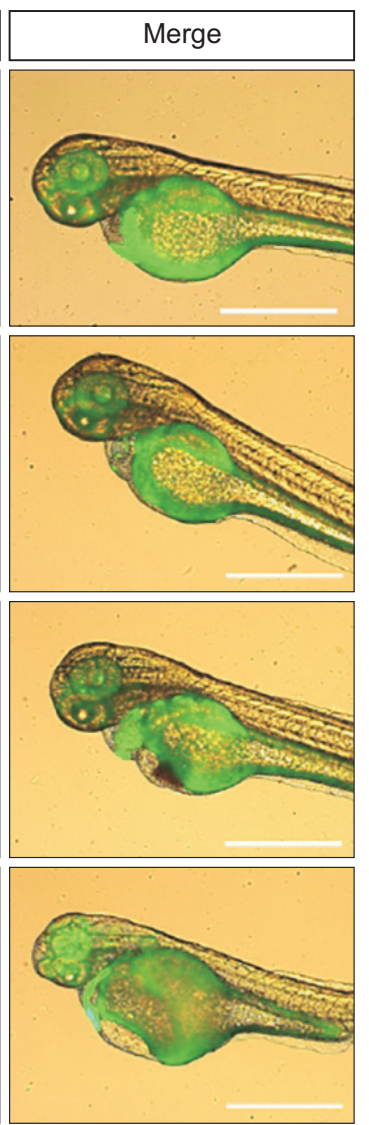

D

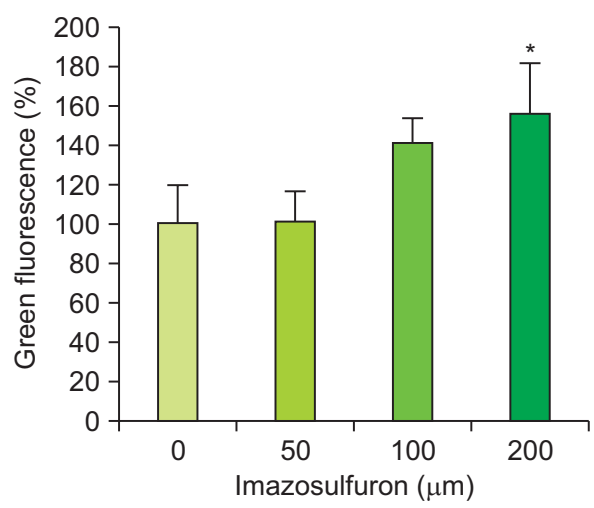

Fig. 2. The lethal effects of imazosulfuron treatment on zebrafish embryos. (A) The dose-dependent viability of zebrafish embryos in response to imazosulfuron treatment. (B) Heartbeats were counted in 48 hpf zebrafish embryos after imazosulfuron treatment. (C) The apoptotic cells in zebrafish embryos were visualized with acridine orange staining and was observed as a green signal. Arrows indicate the apoptotic cells in the anterior part of the embryos. (D) The graph shows relative green fluorescence compared to vehicletreated zebrafish embryos. For these experiments, 20 embryos were used per dosage $\left({ }^{*} p<0.05,{ }^{* *} p<0.01\right.$ and $\left.{ }^{* *} p<0.001\right)$.

logical results including growth failure, edema, or even death might occur (Anderson, 1996). Imazosulfuron treatment of zebrafish embryos induced pericardial edema, and this heart malformation resulted in a decrease in the number of heartbeats. Moreover, imazosulfuron treat- ment induced spine malformation in zebrafish embryos. Early-stage scoliosis hampered tail bending, which is the first step required for zebrafish locomotion and escape responses. This kinked tail is a common result of toxicity on zebrafish embryos (Godoy et al., 2015). Spine develop- 
A
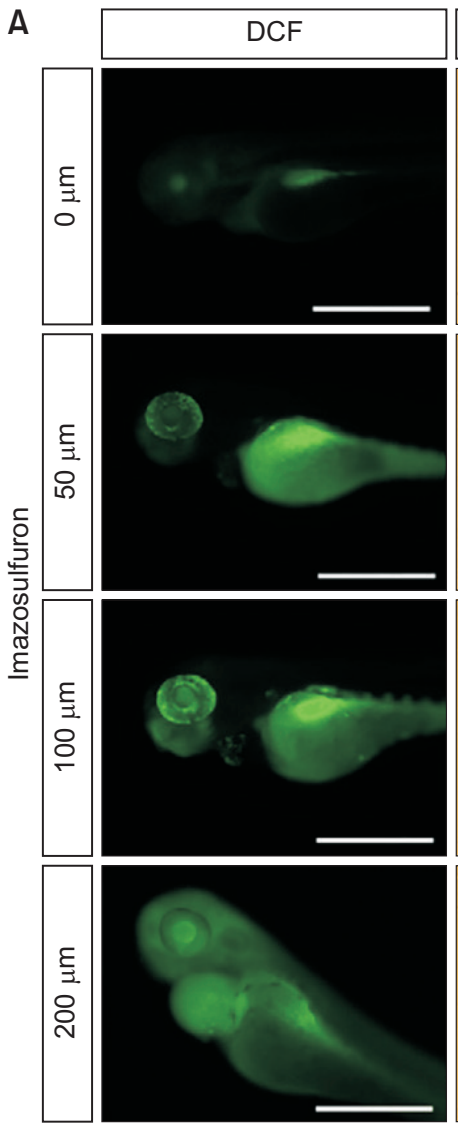
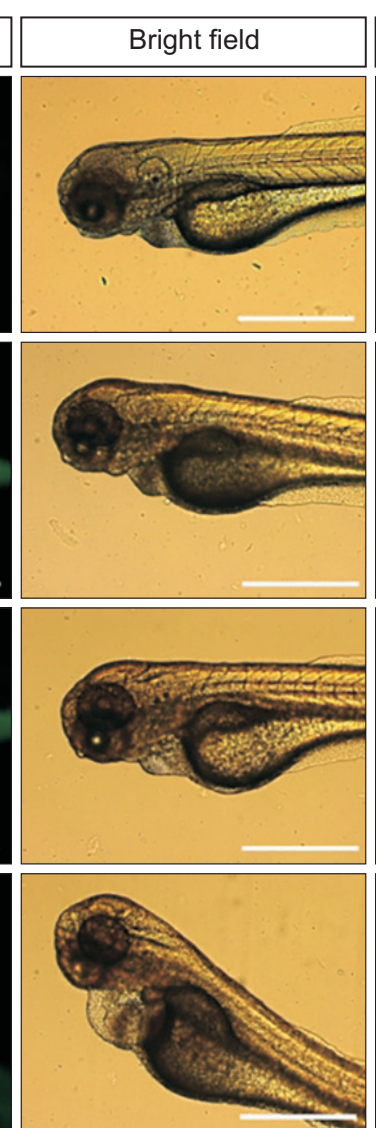
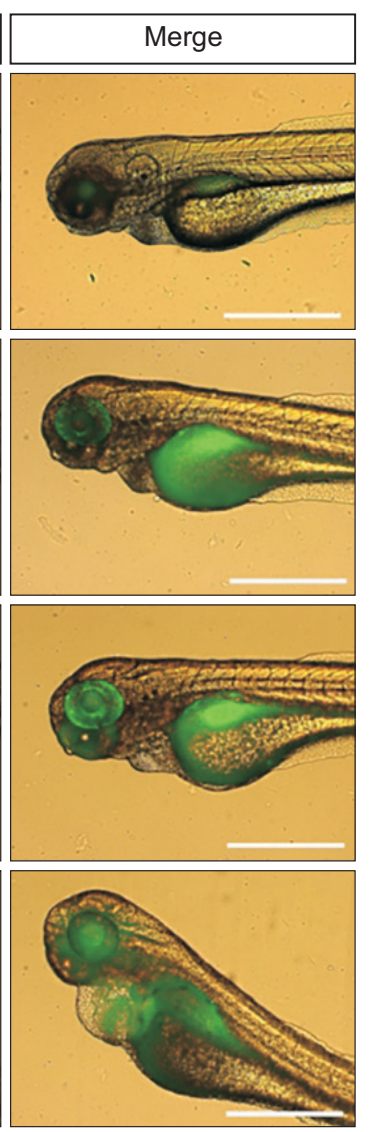

B

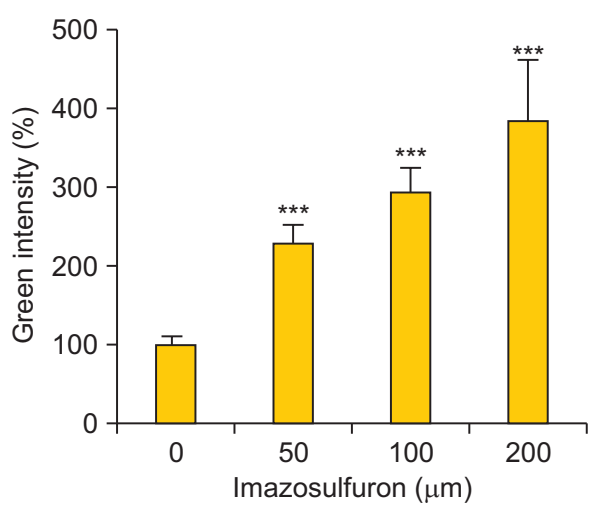

Fig. 3. Oxidative stress on zebrafish embryos in response to imazosulfuron treatment. (A) ROS production was detected as green fluorescence with oxidized DCF in zebrafish embryos. (B) The intensity of green fluorescence is shown as a graph. Scale bar represents 0.5 $\mathrm{mm}$. For these experiments, 20 embryos were used per dosage $\left({ }^{* * *} p<0.001\right)$.

ment is known to be related with kinesin family member 6 (kif6) and protein tyrosine kinase 7 (ptk7) in zebrafish (Buchan et al., 2014; Hayes et al., 2014). Both heart dysfunction and scoliosis in the present study implied that acute dosages of imazosulfuron caused anti-developmental effects of in zebrafish embryos.

Aside from anti-developmental effects, imazosulfuron also gradually decreased the viability and number of heartbeats of zebrafish embryos. We also detected that imazosulfuron induced the apoptosis of embryos, especially in the anterior part and in the eyes and brain. The eyes and brain contain diverse types of glial cells, including optic nerve, retina, neural crest cells, neurons, and astrocytes (Eason et al., 2017). Synthesized organotin compounds induced pyknosis and visual problems with normal retina apoptosis in zebrafish (Biehlmaier et al., 2001). Retina apoptosis decreased retinoic acid synthesis and inhibited the growth and migration of neural crest cells (Matt et al., 2008). In addition, sulfonylurea com- pounds showed high affinity to the axon terminals of neurons in the substantia nigra compared to the cell bodies in the brain (DunnMeynell et al., 1997). Even though we did not characterize each apoptotic cell, apoptosis in the eyes and brain due to imazosulfuron treatment implied that imazosulfuron has lethal toxicity on zebrafish.

It is well known that excessive oxidative stress induces apoptosis in cells. Spontaneous ROS accumulation leads to oxidative stress damage on the body and induces various diseases in the eyes (Kruk et al., 2015). For example, one of the organotin compounds, trimethyltin chloride, dramatically increased ROS generation and histopathological abnormalities in the eyes of zebrafish embryos; this is because eyes are directly exposed organs (Kim et al., 2019). Moreover, sulfonylureas such as glibenclamide, glimepiride, and nateglinide also generate intracellular ROS and lead to apoptosis in pancreatic $\beta$-cells (Sawada et al., 2008). Likewise, imazosulfuron increased ROS generation by $384 \%$ in the eyes compared to normal zebrafish 
embryos. The results from the present study thus suggest that imazosulfuron has anti-developmental effects and can cause lethal toxicity in zebrafish during embryogenesis.

\section{CONCLUSION}

In this study, we discovered the acute toxic effects of imazosulfuron on zebrafish. Imazosulfuron induced malformation of the heart and spine without causing changes in other organs. Furthermore, it induced oxidative stressrelated apoptosis in zebrafish embryos. Thus, the results from this study show, for the first time, the pathological effects of imazosulfuron during zebrafish embryogenesis.

\section{CONFLICTS OF INTEREST}

No potential conflict of interest relevant to this article was reported.

\section{ACKNOWLEDGEMENTS}

This research was supported by a grant of the National Research Foundation of Korea (NRF) grant funded by the Ministry of Science and ICT (MSIT) [grant number 2018R1C1B6009048].

\section{AUTHOR CONTRIBUTIONS}

S.P., G.S.., and W.L. conceived and designed all experiments; S.P. collected experimental samples and conducted all experiments; G.S., and W.L. analyzed, interpreted the data, and contributed to the development of the manuscript. All authors contributed to its critical review and agreed on the final version. All authors have read and agree to the published version of the manuscript.

\section{AUTHOR'S POSITION AND ORCID NO.}

$$
\begin{aligned}
& \text { S Park, Ph.D., } \\
& \text { https://orcid.org/0000-0002-9925-8065 } \\
& \text { G Song, Professor, } \\
& \text { http://orcid.org/0000-0003-2817-5323 } \\
& \text { W Lim, Assistant Professor, } \\
& \text { https://orcid.org/0000-0002-1328-0465 }
\end{aligned}
$$

\section{REFERENCES}

Anderson PA. 1996. The heart and development. Semin. Perinatol. 20:482-509.

Biehlmaier O, Neuhauss SC, Kohler K. 2001. Onset and time course of apoptosis in the developing zebrafish retina. Cell Tissue Res. 306:199-207.

Blair AM and Martin TD. 1988. A review of the activity, fate and mode of action of sulfonylurea herbicides. Pestic. Sci. 22:195-219.

Buchan JG, Gray RS, Gansner JM, Alvarado DM, Burgert L, Gitlin JD, Gurnett CA, Goldsmith MI. 2014. Kinesin family member 6 (kif6) is necessary for spine development in zebrafish. Dev. Dynam. 243:1646-1657.

Dunn-Meynell AA, Routh VH, McArdle JJ, Levin BE. 1997. Lowaffinity sulfonylurea binding sites reside on neuronal cell bodies in the brain. Brain Res. 745:1-9.

Eason J, Williams AL, Chawla B, Apsey C, Bohnsack BL. 2017. Differences in neural crest sensitivity to ethanol account for the infrequency of anterior segment defects in the eye compared with craniofacial anomalies in a zebrafish model of fetal alcohol syndrome. Birth.Defects Res. 109:1212-1227.

Garcia GR, Noyes PD, Tanguay RL. 2016. Advancements in zebrafish applications for 21st century toxicology. Pharmacol. Ther. 161:11-21.

Godoy R, Noble S, Yoon K, Anisman H, Ekker M. 2015. Chemogenetic ablation of dopaminergic neurons leads to transient locomotor impairments in zebrafish larvae. J. Neurochem. 135:249-260.

Haffter P, Granato M, Brand M, Mullins MC, Hammerschmidt M, Kane DA, Odenthal J, van Eeden FJ, Jiang YJ, Heisenberg CP, Kelsh RN, Furutani-Seiki M, Vogelsang E, Beuchle D, Schach U, Fabian C, Nüsslein-Volhard C. 1996. The identification of genes with unique and essential functions in the development of the zebrafish, Danio rerio. Development 123:1-36.

Hay JV. 1990. Chemistry of sulfonylurea herbicides. Pestic. Sci. 29:247-261.

Hayes M, Gao X, Yu LX, Paria N, Henkelman RM, Wise CA, Ciruna B. 2014. ptk7 mutant zebrafish models of congenital and idiopathic scoliosis implicate dysregulated Wnt signalling in disease. Nat. Commun. 5:4777.

Kim J, Kim CY, Oh H, Ryu B, Kim U, Lee JM, Jung CR, Park JH. 2019. Trimethyltin chloride induces reactive oxygen speciesmediated apoptosis in retinal cells during zebrafish eye development. Sci. Total Environ. 653:36-44.

Kruk J, Kubasik-Kladna K, Aboul-Enein HY. 2015. The role oxidative stress in the pathogenesis of eye diseases: current status and a dual role of physical activity. Mini Rev. Med. Chem. 16:241-257.

Kuk YI, Kim KH, Kwon OD, Lee DJ, Burgos NR, Jung S, Guh JO. 2004. Cross-resistance pattern and alternative herbicides for Cyperus difformis resistant to sulfonylurea herbicides in Korea. Pest Manag. Sci. 60:85-94. 
LaRossa RA and Schloss JV. 1984. The sulfonylurea herbicide sulfometuron methyl is an extremely potent and selective inhibitor of acetolactate synthase in Salmonella typhimurium. J. Biol. Chem. 259:8753-8757.

Li K, Wu JQ, Jiang LL, Shen LZ, Li JY, He ZH, Wei P, Lv Z, He MF. 2017. Developmental toxicity of 2,4-dichlorophenoxyacetic acid in zebrafish embryos. Chemosphere 171:40-48.

Lieschke GJ and Currie PD. 2007. Animal models of human disease: zebrafish swim into view. Nat. Rev. Genet. 8:353-367.

Matt N, Ghyselinck NB, Pellerin I, Dupé V. 2008. Impairing retinoic acid signalling in the neural crest cells is sufficient to alter entire eye morphogenesis. Dev. Biol. 320:140-148.

Morrica P, Barbato F, Giordano A, Seccia S, Ungaro F. 2000. Adsorption and desorption of imazosulfuron by soil. J. Agric. Food Chem. 48:6132-6137.

Morrica P, Giordano A, Seccia S, Ungaro F, Ventriglia M. 2001. Degradation of imazosulfuron in soil. Pest Manag. Sci. 57:360-365.

Moura MAM, Oliveira R, Jonsson CM, Domingues I, Soares AMVM, Nogueira AJA. 2018. The sugarcane herbicide ametryn induces oxidative stress and developmental abnor- malities in zebrafish embryos. Environ. Sci. Pollut. Res. Int. 25:13416-13425.

Pickart MA and Klee EW. 2014. Zebrafish approaches enhance the translational research tackle box. Transl. Res. 163:65-78.

Sawada F, Inoguchi T, Tsubouchi H, Sasaki S, Fujii M, Maeda Y, Morinaga H, Nomura M, Kobayashi K, Takayanagi R. 2008. Differential effect of sulfonylureas on production of reactive oxygen species and apoptosis in cultured pancreatic betacell line, MIN6. Metabolism 57:1038-1045.

Sondhia S. 2008. Determination of imazosulfuron persistence in rice crop and soil. Environ. Monit. Assess. 137:205-211.

Villeneuve D, Volz DC, Embry MR, Ankley GT, Belanger SE, Léonard M, Schirmer K, Tanguay R, Truong L, Wehmas L. 2014. Investigating alternatives to the fish early-life stage test: a strategy for discovering and annotating adverse outcome pathways for early fish development. Environ. Toxicol. Chem. 33:158-169.

Wang B, Kong D, Lu J, Zhou Q. 2015. Transformation of sulfonylurea herbicides in simulated drinking water treatment processes. Environ. Sci. Pollut.Res. Int. 22:3847-3855. 